\title{
Wissensobjekt und Sinnformel Selbstmordattentat
}

\author{
Über den Zusammenhang von politischer und epistemischer Gewalt \\ am Beispiel gegenwärtiger Terrorismusforschung
}

Dieser Aufsatz fasst die Ergebnisse einer Forschungsarbeit zusammen, in der Texte, Bilder und Grafiken aus zentralen Werken der englischsprachigen Terrorismusforschung (ca. 1996-2007) einer wissenssoziologisch-diskursforschenden Analyse unterzogen wurden. Integriert in eine postkolonial-feministisch verortete Perspektive wird ein Zusammenhang zwischen politischer und epistemischer Gewalt innerhalb global asymmetrischer Machtverhältnisse argumentiert, in denen sich nicht nur die beforschten Attentate ereignen, sondern auch die Forschung selbst stattfindet. Im (sozial-)wissenschaftlichen Wissensobjekt Selbstmordattentat verdichten sich demnach Zuschreibungen von Eigen-heit und Andersheit, die nicht nur über Gegenstand und AkteurInnen Auskunft geben. Vielmehr werden an ihm auch die Konturen, Horizonte und Ambivalenzen von Terrorismusforschung sichtbar. Spezifisch ideologisiert und generalisiert steht dieses wissenschaftliche Wissensobjekt auch in Beziehung zu außerwissenschaftlichen Wissensbeständen und wird damit bisweilen zu einer Sinnformel in einem vermeintlichen »Kampf der Kulturen«. Zu dieser gegenwärtig allzu plausibel erscheinenden Kulturalisierung des Politischen trägt auch jene wissenschaftliche Expertise bei, die den Gegenstand der hier vorgestellten Arbeit bildet: Terrorismusforschung.

Schlagworte: Selbstmordattentat, Wissen, Okzidentalismus, epistemische Gewalt, Terrorismus

»Jenseits affirmativer Forschung, die in ihren Analysen bestehende Deutungen zusätzlich stützt, sollte das kritische Hinterfragen gesellschaftlich anerkannter Definitionen von Wirklichkeit das Kernanliegen unabhängiger Sozial- und Kulturwissenschaften bleiben. Nichts sollte sie von dieser Art des Denkens und Forschens abhalten, vor allem nicht über den Stoff, aus dem diese Disziplinen selbst gewoben sind: dem Wissen.« (Kajetzke 2008: 162)

\section{Einleitung, Verortung und Überblick}

Die von mir untersuchte englischsprachige, als Mainstream zu bezeichnende Terrorismusforschung der letzten Jahrzehnte trägt entscheidend dazu bei, eine ganz 
spezifische suicide terrorism story als ordnenden Diskurs in der Auseinandersetzung um die (Il-)Legitimität von politischer Gewalt zu etablieren. ${ }^{1}$ Dieser geht über das spezialisierte Feld der Terrorismusforschung selbst hinaus und mit einer gegenwärtigen okzidentalistischen ${ }^{2}$ Selbstvergewisserung dominanter Positionen im global asymmetrischen Gefüge von Macht, Gewalt und Herrschaft einher, das sich nach dem Ende der bipolaren Konstellation 1989 sowie den Anschlägen auf Pentagon und World Trade Center 2001 neu organisiert. Die dabei entstehende ordnungs- und sinnstiftende dominante Narration im hegemonialen Terrorismuswissen rund um das sich herausbildende Wissensobjekt Selbstmordattentat ist von Partikularismen und Generalisierungen durchzogen, die oft mehr mit den epistemischen und politischen Voraussetzungen und Konsequenzen hegemonialer Terrorismusforschung zu tun haben als mit den konkreten Ereignissen, die diese zu erklären beansprucht.

Die Diskussion von und Kritik an Forschung zu einer Gewaltpraxis im Spannungsfeld globaler Asymmetrien und Ungleichheitsverhältnisse wird dabei in vielerlei Hinsicht vom Thema Gewalt durchquert. Direkte und physische politische Gewalt bilden den Gegenstand der hier diskutierten Forschung selbst; strukturelle Gewalt durchzieht den Rahmen, innerhalb dessen diese Forschung betrieben wird, wie auch jenen, der die von ihr begleitete Terrorismusbekämpfung hervorbringt. Mit dem Begriff der epistemischen Gewalt (Spivak 1988; 2008) fokussiere ich auf die Bedingungen und Konsequenzen von Produktion, Distribution und Rezeption von wissenschaftlicher Expertise zu Terrorismus und politischer Gewalt. Wissen wird hier also nicht als getrennt und unabhängig von politischen Macht-, Gewaltund Herrschaftsverhältnissen, sondern als dazu relational verstanden - und ins Zentrum des Erkenntnisinteresses gerückt. Nach Gayatri C. Spivak ist »epistemische Gewalt [...] das aus der Distanz orchestrierte, weitläufige und heterogene Projekt, das koloniale Subjekt als Anderes zu konstituieren« (Spivak 2008: 42) und damit die Position dominanter Erklärungs- und Handlungsweisen zu stärken. Dass wissenschaftliche Expertise an diesen Prozessen maßgeblich beteiligt ist, ist spätestens

1 Basis dieses Aufsatzes ist die Arbeit »Sinnformel Selbstmordattentat« (Dissertation) bzw. »Wissensobjekt Selbstmordattentat « (Publikation; Brunner 2011 a). Sie entstand 2005 bis 2008 im Rahmen des von der Deutschen Forschungsgemeinschaft geförderten Graduiertenkollegs »Geschlecht als Wissenskategorie« am Zentrum für Transdisziplinäre Geschlechterstudien der Humboldt-Universität zu Berlin. Die Arbeit wurde 2009 am Institut für Politikwissenschaft der Universität Wien verteidigt und 2011 mit dem Christiane Rajewsky-Preis der Arbeitsgemeinschaft für Friedens- und Konfliktforschung ausgezeichnet. Für kritische Lektüren und Kommentare früherer Versionen dieses Textes danke ich Mechthild Exo, Magdalena Freudenschuß und Kerstin Piepenstock sowie den anonymen GutachterInnen der ZeFKo.

2 Mehr zum Begriff des Okzidentalismus nach Coronil (1996; 2002) siehe Abschnitt 2.3. 
seit Edward Saids Orientalism (1978) unbestritten. Man kann Wissenschaft demnach als potenziellen »Herrschaftsdienst « (Pappe 2011) verstehen, der selbst in seiner kritischen und widerständigen Variante nicht jenseits von politischen Machtund Gewaltverhältnissen betrieben und auch nicht vollständig verweigert werden kann. Die darin eingelagerte epistemische Gewalt, die unser Wissen über politische Gewalt mit hervorbringt, verstehe ich in einem konstitutiven Zusammenhang mit jenen Formen politischer Gewalt, deren wissenschaftliche Analysen sich im sozialwissenschaftlichen Wissensobjekt Selbstmordattentat zu zunehmend normalisiertem Terrorismuswissen verdichten.

Die deutschsprachige Friedens- und Konfliktforschung unterscheidet sich gewiss in vielerlei Hinsicht vom Untersuchungsgegenstand meiner Arbeit, der englischsprachigen Terrorismusforschung. In manchen Punkten jedoch kann ein Überdenken der potenziellen Verschränkungen zwischen politischer Gewalt, die wir analysieren, und epistemischer Gewalt, die unsere Forschungsperspektiven ebenso beeinflusst wie die Rahmenbedingungen, unter denen diese erst hervorgebracht werden können, am Beispiel des Wissensobjekts Selbstmordattentat angeregt werden. ${ }^{3}$ Selbstverständlich bin auch ich selbst mit meiner Wissensproduktion in diesem Setting positioniert. Zwar ist mein Beitrag zur wissenschaftlichen Debatte sowohl aus sprachlichen (nicht im englischen Mainstream, daher von diesem kaum rezipiert) als auch aus epistemologischen Gründen (dekonstruktive Perspektive, die mit positivistischer Terrorismusforschung kaum kompatibel ist) bestenfalls an einem der Ränder des von mir untersuchten Feldes zu verorten. Nichtsdestotrotz interveniert diese Perspektive z.B. auch in Form des vorliegenden Beitrags in die hier problematisierte Herausbildung des Wissensobjekts Selbstmordattentat. Diesen Widerspruch explizit und durch methodologische und epistemologische Reflexion bzw. Darlegung der eingenommenen SprecherInnenposition transparent zu machen, stellt eine Möglichkeit dar, einen selbstreflexiven Umgang mit der eigenen Verortung im Gefüge wissenschaftlicher Wissensproduktion - und nicht zuletzt mit der von mir problematisierten epistemischen Gewalt, die dieser innewohnen kann - zu pflegen. Dass dies insbesondere von jenen AutorInnen immer wieder eingefordert wird, deren Arbeiten nicht selbstverständlich mit dem jeweiligen Mainstream kompatibel sind, wohingegen selbstverständlicher erscheinende Positionierungen darin kaum zu aktiver Selbstreflexion aufgefordert werden, ist Bestandteil der Kanonisierung, Normalisierung und Marginalisierung von Wissen. Ich komme der in einem

3 In Bezug auf die institutionelle Dimension, die mit der epistemischen auf das Engste verknüpft ist, siehe Exo (2009); zu materiellen und forschungspraktischen Implikationen etwa zu Hochschulforschung für militärische Zwecke siehe auch Nagel (2009). 
Gutachten zu diesem Beitrag formulierten Aufforderung zur Selbstpositionierung hiermit gerne nach. Zugleich ist mir wichtig, darauf hinzuweisen, dass die Verstrickung in Dimensionen epistemischer Gewalt keineswegs nur auf das Individuum, das sprechende/schreibende Subjekt, den/die AutorIn beschränkt ist. Vielmehr geht es dabei um tiefliegende symbolisch-kulturelle und weit verbreitete politisch-institutionelle Verankerungen und Einlassungen in komplexen Settings von Wissen und Macht. Mit der Kritik an Konventionalisierungen der hier untersuchten Wissensbestände will ich also keine Handlungsanweisung für eine bessere Terrorismusforschung vorlegen, sondern diese selbst in ihren Standorten und Standpunkten zwischen Forschung, Politikberatung und Terrorismusbekämpfung herausfordern. Das mag auf den ersten Blick defensiv erscheinen, ist im Sinne der hier dargelegten Perspektive jedoch nur konsequent und schlüssig. Einige Ergebnisse daraus können auch für die in dieser Zeitschrift künftig geführten Diskussionen um die adäquate Beforschung von Frieden und Konflikten als Bausteine einer wissenssoziologischen Grundlagenforschung verstanden und genützt werden.

Dieser Aufsatz gliedert sich in vier Abschnitte. Zunächst werden mit der Erläuterung von Gegenstand, Fragestellung, Perspektive, Material und Methoden das Erkenntnisinteresse der Untersuchung sowie deren theoretische und epistemologische Verortung explizit gemacht (2). Die verdichtete Darstellung der Analyseergebnisse erfolgt in drei weiteren Teilen: Darin werden jeweils unterschiedliche Ebenen der Entstehung des Wissensobjekts bzw. Dimensionen der »Sinnformel« (Geideck/Liebert 2003) Selbstmordattentat diskutiert: Die Konturen des Wissensobjekts verweisen auf Entstehung, Definitionspräferenzen und Bekämpfungsperspektive in einem sich gleichzeitig als eigenständige Disziplin herausbildenden Feld der Terrorismusforschung (3). In den Wegen zum Wissen wird der Zusammenhang von methodischen, theoretischen, epistemologischen und schließlich politischen Fragen rund um den Gegenstand diskutiert (4). Die kursorisch dargelegten sechs Modi der okzidentalistischen Selbstvergewisserung - Pathologisierungen, (Ir-) Rationalisierungen, Sexualisierungen, Historisierungen, Geopolitisierungen und Kulturalisierungen - zeigen schließlich die vielschichtigen diskursiven Prozesse, in denen das Wissensobjekt zur Sinnformel geworden ist (5). Ein zusammenfassender Ausblick (6) rundet den Text ab.

\section{Forschungsdesign}

»Auf Gewalt zu zeigen heißt immer, Machtverhältnisse zur Diskussion zu stellen«, so Carol Hagemann-White (2002: 29). Die vorgestellte Arbeit versteht sich als Beitrag zu diesem immer wieder erforderlichen Unterfangen. Es geht mir darum, am 
Beispiel des Wissensobjekts Selbstmordattentat in der Terrorismusforschung die in Bezug auf epistemische Gewalt relevanten Bedingungen und Konsequenzen des Wissenschaft-Betreibens selbst zur Diskussion zu stellen. Ein solches Vorhaben erfordert konzeptionelle Klärungen in epistemologischer, theoretischer, methodologischer und methodischer Hinsicht. Diese erläutere ich in vier Schritten: Definition des Gegenstandes, Begründung der Fragestellung, Darlegung des theoretischen Zugangs sowie schließlich Benennung von Material und Arbeitsweisen. ${ }^{4}$

\subsection{Gegenstand: Das Wissensobjekt Selbstmordattentat im hegemonialen Diskurs der Ver(un-)sicherheitlichung}

Innerhalb der Internationalen Beziehungen ${ }^{5}$ und quer zu diesen etablierte sich in den vergangenen zwei Jahrzehnten nicht nur ein Forschungsfeld, sondern auch eine Subdisziplin der Terrorismusforschung. Das Wissensobjekt Selbstmordattentat, so meine These, hat sich darin als eigenständiger Gegenstand herausgebildet und zur Konstituierung und Kanonisierung von hegemonialem Terrorismuswissen dynamisch beigetragen. Drei Elemente kennzeichnen dieses Feld in besonderer Weise. Erstens kann die Terrorismusforschung als Teil eines Sicherheitsdispositivs im Sinne Michel Foucaults (1978) verstanden werden, das ein gouvernementales (Foucault 2000) Eigenleben entwickelt hat. Nationale und supranationale Gewaltmonopole, die unter anderem von zunehmend im Begriff des Selbstmordterrorismus vereindeutigten Anschlägen in Frage gestellt werden, nutzen deren Bekämpfung ebenso wie deren Beforschung zur Regeneration ihrer Macht (Holert 2004: 247). Damit ist zweitens die Sonderrolle von wissenschaftlichem ExpertInnenwissen angesprochen, das im Fall des hier untersuchten Gegenstandes auf soliden eurozentrischen Grundannahmen und Praktiken beruht. Dieses Wissen verfügt nicht nur über einen erhöhten Wahrheitsanspruch, der die ihm innewohnenden asymmetrischen Machtverhältnisse verdeckt (Elshtain 1987: 91), sondern kann auch institutionell wie personell oft nahe an politischen und militärischen (Diskurs-)Eliten verortet werden. Darüber hinaus weist dieses Wissen auch dort, wo dies nicht der Fall ist, eine hohe interdiskursive Anschlussfähigkeit in Bezug auf das Kernelement der später ${ }^{6}$ noch auszuführenden okzidentalistischen Selbstvergewisserung auf. Das »Phänomen Selbstmordattentat« bietet hierfür einen geradezu idealen Kristallisationspunkt, an

4 Im Rahmen dieses Aufsatzes können nicht alle Begründungen und Vorgangsweisen detailliert angeführt werden. Zum gesamten Forschungsdesign und den hier kursorisch angedeuteten vier Ebenen der Konzeption siehe Brunner (2011 a: 21-79).

5 Damit meine ich sowohl die wissenschaftliche Disziplin als auch die politische Praxis.

6 Siehe Abschnitt 2.3. 
dem viele Facetten der Herstellung von Selbst-Verständlichkeit und Eigen-heit ${ }^{7}$ durch die angestrebte maximale Abgrenzung gegenüber einem auf mehrfache Weise "ganz anderen Anderen" (Sarasin 2003: 49) zusammentreffen. Dies führt mich drittens zur Sinnformelhaftigkeit des allmählich entstehenden Wissensobjekts Selbstmordattentat. Mit »Sinnformel« meinen Susan Geideck und Wolf-Andreas Liebert (2003) einen diskursiven Knotenpunkt, in dem sich Grundfragen nach Identität, Geschichte, Gegenwart und Zukunft verdichten. Dynamisch in ihrem Charakter und mit einem starken imaginativen Kern, so Geideck und Liebert, bewegen sich Sinnformeln zwischen Nicht-Thematisierung und Kontroverse. Je weniger sie hinterfragt werden, umso effizienter funktionieren sie und umso weniger sind sie den an ihrer (Re-)Produktion Beteiligten zugänglich (Geideck/Liebert 2003: 3-8). Dies trifft auf das wissenschaftliche Wissensobjekt Selbstmordattentat in vielfacher Weise zu.

\subsection{Fragestellung: Der Wille zum Wissen über das (Nicht-)Wissenwollen}

Wie wird in aktuellen sozialwissenschaftlichen Debatten innerhalb hegemonialer Wissensbestände ein Wissensobjekt Selbstmordattentat hervorgebracht? Auf welchen Wegen und mit welchen Ergebnissen geschieht dies? Welche Vorstellungen von Legitimität und Illegitimität politischer Gewalt werden dabei verhandelt? Und wie funktionieren Einschlüsse und Ausschlüsse von Wissen im Verlauf von zwanzig Jahren Terrorismusforschung zum Thema Selbstmordattentat?

Gewalt ist nicht die Ausnahme von der Regel der Internationalen Beziehungen, sondern vielmehr für diese konstitutiv. Unter Gewalt verstehe ich ein dichtes Geflecht unterschiedlicher Dimensionen, das strukturelle (Galtung 1975) und symbolische (Bourdieu 1982) Gewalt mit einschließt. Nicht dass wissenschaftliches Wissen über Selbstmordattentate produziert wird, will ich problematisieren, sondern wie und wozu dies geschieht, leitet mein Erkenntnisinteresse. Über die Stufe des Zweifelns (vgl. Hitzler 2002: 26) hinausgehend und eine Konkretisierung einer neuen Perspektive auf das komplexe Verhältnis von Wissen und Macht vorschlagend orientiere ich mich dabei zentral am Begriff der epistemischen Gewalt nach Gayatri C. Spivak (1988; 2008: 42) und stelle diesen in einen Zusammenhang mit jenen Formen von Gewalt, die in der Terrorismusforschung davon gänzlich isoliert erscheint. Spivaks Kritik an eurozentrischer Wissensproduktion erschüttert politische und epistemologische Gewissheiten. Sie zeigt deutlich, dass Wissenschaft im

7 Diese Schreibweise ist beabsichtigt, um die Herstellung des Selbst, des Eigenen, zu betonen, die diesen Prozessen innewohnt. Genau darum geht es im Konzept des Okzidentalismus nach Fernando Coronil $(1996 ; 2002)$. 
Kontext post- und neokolonialer Machtverhältnisse weder per se objektiv noch aufklärend ist, sondern vielmehr ein wandlungs- und tragfähiges Netz für die Befestigung von Dominanz und Ungleichheit im imperialen, neoliberalen, kapitalistischen Weltsystem darstellt. Die konkreten Anschläge stellen also nicht nur eine politische, sondern für die Sozialwissenschaften auch eine epistem(olog)ische Herausforderung dar. Die Konstituierung des Wissensobjekts Selbstmordattentat, so wie es sich im Laufe von 20 Jahren Terrorismusforschung entwickelt hat, befestigt die grundlegende Trennung eben jener Bereiche, die ich am Beispiel des Wissensobjekts Selbstmordattentat zusammendenken will: politische und epistemische Gewalt. Darüber hinaus kann mit diesem Zugriff eine analytische Distanz zu einem komplexen Phänomen der Gegenwart (damit meine ich unterschiedliche als Selbstmordattentate/Selbstmordterrorismus bezeichnete Anschläge) sowie zu dessen diskursiver Herstellung (damit meine ich die Vereindeutigung unseres Wissens darüber) hergestellt werden, um grundsätzlicher über Terrorismuswissen nachzudenken, als die damit befasste Forschung selbst dies tut. Erst dann kann der Blick für den globalen Kontext asymmetrischer Machtkonstellationen, Wissensproduktionen und Wahrheitsregime geweitet werden, in dem Selbstmordattentate nicht nur verübt, sondern auch beforscht und bekämpft werden. Eine solche Perspektive kann auch der Friedens- und Konfliktforschung neue Impulse geben. Sie knüpft sie an deren kritische Anfänge an und übersetzt sie in die Gegenwart, indem sie ihr transdisziplinäres Potenzial $\mathrm{zu}$ anderen kritischen wissen(schaft)stheoretischen Traditionen ausschöpft, die im Folgenden vorgestellt werden.

\subsection{Perspektive: Wissenssoziologische Diskursanalyse, Feministische Intersektionalitätsforschung und Okzidentalismuskritik}

Für die Entwicklung einer adäquaten Theoretisierung dieser Perspektive kombiniere ich drei unterschiedliche Theorietraditionen, die bislang nicht systematisch miteinander verwoben worden sind. Es sind dies erstens die Wissenssoziologische Diskursanalyse nach Reiner Keller (2004; 2005), in methodischer Hinsicht ergänzt durch Teile der Kritischen Diskursforschung (Jäger 1999; Fairclough 1995), zweitens Postkoloniale Theorie, insbesondere das Konzept der Okzidentalismuskritik (Coronil 1996; 2002), das auf Edward Saids Kritik am Orientalismus (1978) basiert und dieses weiterentwickelt, und drittens die explizit feministische Verortung ${ }^{8}$ dieser Zugänge. Eine weitere Grundlage dieser Perspektivierung bildet die Intersek-

8 Für die Postkoloniale Theorie vgl. Spivak (1988; 2008); Yeğenoğlu (1999); Lewis/Mills (2003); Dietze et al. (2009); für die Internationalen Beziehungen vgl. Elshtain (1987); Whitworth (1997); Sylvester (2002); Enloe (1989; 2004). 
tionalitäts- und Interdependenzforschung. ${ }^{9}$ Nach Melisa Brittain (2006: 93) muss sich eine feministische Perspektive, die auf die Analyse von Sinnproduktion abzielt, an der intersektionalen Verwobenheit von Rasse, Geschlecht, Klasse und Sexualität abarbeiten, je nach Gegenstand und Erkenntnisinteresse ergänzt durch weitere Kategorien. Nur dann kann sie in die großen Narrative, in einem war on terror und darüber hinaus intervenieren, die imperialistische, rassisierte, vergeschlechtlichte und sexuelle Gewalt immer wieder bestätigen (Brittain 2006: 22). Neben dem analytischen Potenzial des Konzepts Intersektionalität ist es vor allem ein solcher herrschaftskritischer und politischer Impetus, der es für meine Forschungsperspektive nützlich macht. Darüber hinaus ist von intersektionalen Zugängen auch eine mögliche Verbindung zwischen kleinteiligen Untersuchungen und großrahmigen gesellschaftlichen Zusammenhängen zu erwarten, also eine gewisse Aussagefähigkeit auf der Meso-Ebene sozialwissenschaftlicher Forschung (Knapp 2005: 71), worauf auch die Wissenssoziologische Diskursforschung abzielt. Es handelt sich also bei Intersektionalitäts- und Interdependenzforschung wie auch bei Okzidentalismuskritik und Wissenssoziologischer Diskursanalyse und nicht zuletzt bei einer feministischen Verortung innerhalb der Friedens- und Konfliktforschung um theoretische Programmatiken mit politischem Anspruch, die gesellschaftstheoretisch anschlussfähig gemacht werden können. Genau das beabsichtigt mein Versuch, politische und epistemische Gewalt zusammenzudenken.

Um die bereits erwähnte okzidentalistische Selbstvergewisserung im Terrorismuswissen näher zu erläutern, möchte ich nun auf den Begriff des Okzidentalismus nach Fernando Coronil eingehen. ${ }^{10}$ Darunter versteht der Autor

»all jene Praktiken der Repräsentation, die an der Produktion von Konzeptionen der Welt beteiligt sind, welche (1) die Komponenten der Welt in abgegrenzte Einheiten unterteilen; (2) ihre relationalen Geschichten voneinander trennen; (3) Differenz in Hierarchie verwandeln; (4) diese Repräsentationen naturalisieren; und so (5) an der Reproduktion existierender asymmetrischer Machtbeziehungen, und sei es noch so unbewußt, beteiligt sind « (Coronil 2002: 186).

Okzidentalismus kann also als ein wesentliches Paradigma der von Shalini Randeria (1999) so genannten »geteilten Geschichten/entangled histories« bezeichnet wer-

9 Vgl. Collins (1990); Crenshaw (1995); Knapp (2005); McCall (2005); Walgenbach et al. (2007).

10 In den Tagen der Erstellung der ersten Version dieses Aufsatzes im August 2011 ist der venezolanische Sozialanthropologe und Historiker Fernando Coronil verstorben. Aus diesem Anlass möchte ich ihm meinen Text widmen und dazu anregen, seine weitsichtigen Thesen auch im deutschsprachigen Raum weiter zu diskutieren. Siehe auch Coronil (2000; 2009). 
den, die dazu dienen, bestehende Machtasymmetrien und Herrschaftsverhältnisse aufrechtzuerhalten. Dieser Fokus ist es, den gerade auch eine feministische, postkoloniale und intersektionale Perspektive sowie deren Verschränkung mit der Wissenssoziologischen Diskursanalyse bestens zu untersuchen geeignet ist. $\mathrm{Zu}$ einer solchen Positionierung zählt auch das Explizitmachen von und Reflektieren über Material und Methoden, die im nächsten Abschnitt vorgestellt werden.

\subsection{Material und Methoden: Kohärenter Umgang mit heterogenen Manifestationen von Terrorismuswissen}

Texte, Bilder, Grafiken, Modelle und Paratexte ${ }^{11}$ treten Lesenden und Forschenden als Collage entgegen, wobei nicht-textliches Material, das sich innerhalb von und rund um verschriftlichtes ExpertInnenwissen befindet, bislang noch keine systematische, methodologische Aufmerksamkeit in der Diskursforschung erfährt. ${ }^{12}$ Mit gerade diesem - Grafiken und Modelle sowie Umschlagbilder, Illustrationen und paratextliche Elemente - habe ich meine Analysen begonnen. Der weitaus überwiegende Teil des zu analysierenden Materials jedoch bestand aus Texten aus dem Feld der englischsprachigen sozialwissenschaftlichen Terrorismusforschung (ca. 1996 bis 2007), die als Zeitschriftenartikel, Beiträge zu Sammelbänden oder Monografien publiziert wurden und aufgrund ihrer institutionellen, personellen, inhaltlichen oder rezeptionsbezogenen Signifikanz für die Herausbildung des Wissensobjekts als relevant erachtet werden können. ${ }^{13}$ Vorgegeben vom Material selbst

11 Paratextliche Elemente sind nach Gérard Genette (2001) etwa Bildunterschriften, Indizes, Vorwörter, Fußnoten, aber auch produktions- und vermarktungsspezifische Hinweise wie das Impressum, Referenzen auf Buchrücken etc.

12 Für die deutschsprachige Debatte bietet der Band von Keller und Truschkat (i.E.) einen ersten Überblick über Erweiterungen der bislang stark text- und sprachorientierten Diskursforschung in Richtung visueller Analysen. Vgl. auch Fegter (2011).

13 Die Basis des untersuchten Materials besteht aus verschriftlichten Analysen und Berichten in unterschiedlichen Publikationsformen. Dazu gehören Monografien (O’Neill 1981; Davis 2003; Israeli 2003; Laqueur 2003; Victor 2004; Reuter 2004; Shay 2004; Bloom 2005; Khosrokhavar 2005; Pape 2005; Pedahzur 2005; Skaine 2006; Berko 2007; Hafez 2007), Anthologien (Reich 1990; ICT 2001; ICT/ADL 2002; Gambetta 2005; Pedahzur 2006) und daraus ausgewählte Beiträge (Merari 1990; Ganor 2002 a; 2002 b; Elster 2005; Ricolfi 2005; Biggs 2005; Holmes 2005; Alonso/Reinares 2006; Hafez 2006; Moghadam 2006 a; 2006 b; Schweitzer 2002; 2006; Weinberg 2006). Des Weiteren wurden wissenschaftliche Artikel in Fachzeitschriften des Spezialdiskurses zu Terrorismusforschung berücksichtigt, insofern sie öffentlich zugänglich sind, nämlich Terrorism and Political Violence (Israeli 1997; Weinberg et al. 2003; Strenski 2003; Kimhi/Even 2004; Pedahzur 2004; Silke 2006; Lewis 2007), Studies in Conflict and Terrorism, später Conflict and Terrorism Studies genannt (Kushner 1996; Hoffman 2002; Cunningham 2003; Dolnik 2003; Moghadam 2003; Hoffman/McCormick 2004; Lester et al. 2004) sowie weitere vereinzelte Beiträge in wissenschaftlichen 
wurde der Untersuchungszeitraum festgelegt. In Fachzeitschriften der englischsprachigen Terrorismus- und Gewaltforschung wurde ab Mitte der 1990er Jahre zum Thema Selbstmordattentat publiziert, eine Häufung und Verdichtung fand in Zeitschriften ab 2002/2003, in Buchform ab 2003 statt. Beide Formate konnten bis zum Erscheinungsjahr 2007 für die Analyse berücksichtigt werden. In Hinblick auf die dispositive Dimension des Untersuchungsgegenstandes wurde insbesondere berücksichtigt, welche Verlage, Institutionen oder Forschungsprojekte, Politikberatungsinstanzen und auch WissensakteurInnen - in ihren Funktionen im Wissensbetrieb - in der Wissensherstellung involviert sind, welche führenden Fachzeitschriften benannt werden können, auf welche gegenseitigen Rezeptionen und Empfehlungen man stößt; kurz: an welchen Parametern sich im Wissenschaftsbetrieb und in damit verbundenen öffentlichen Debatten die Generierung und das Bedienen von symbolischem und sozialem Kapital (Bourdieu 1982) innerhalb dieser diskursiven Praxisfelder ermessen lässt. Geleitet von der Forschungshaltung der Grounded Theory (Glaser/Strauss 1998) und mit Hinblick auf die Entwicklung eines diskursanalytischen Vorgehens hin zu einem dispositiv-analytischen Verständnis des Materials, das AkteurInnen, Institutionen und Strukturen des Wissens verstärkt berücksichtigt (Bührmann/Schneider 2008), wurde im Verfahren des offenen Kodierens nach Barney Glaser und Anselm Strauss (1998) ein breit angelegter Materialkorpus von Texten aus Fachzeitschriften, Monografien und Anthologien definiert, durch den ich mich in wissenssoziologisch-diskursforschender Lesart (Keller 2005) in einem ersten Durchgang chronologisch hindurchgearbeitet habe. Aus den daraus resultierenden thematisch kategorisierbaren Ergebnissen wurde durch axiales Kodieren (Glaser/Strauss 1998) ein verkleinertes Materialdossier zusammengestellt, das ich für detaillierte Analysen herangezogen habe. Diese erfolgten je nach Materialsorte mit spezifischen Methoden der sozialwissenschaftlich orientierten Textund Bildanalyse, die auf diesem Wege in das Programm der Wissenssoziologischen Diskursforschung nach Keller (2005) integriert werden konnte. ${ }^{14}$ Für Texte waren dies insbesondere Jäger (1999: 171-187), Keller (2004: 257-272) und Titscher et al. (1998); für Grafiken und Modelle Kress/van Leeuwen (1996) und Morgan/Morisson

Zeitschriften wie Journal of Conflict Resolution (Dale 1988; Ferrero 2006), Foreign Policy (Sprinzak 2000), Science (Atran 2003), American Political Science Review (Pape 2003), und Artikel aus Zeitschriften, die sich an ein breiteres Publikum zwischen Wissenschaft und Politik richten wie etwa The Atlantic Monthly (Hoffman 2003; Lewis 2002) und The Washington Quarterly (Atran 2006). Zu den institutionellen Verortungen der genannten Verlage und Zeitschriften sowie AutorInnen siehe detailliert Brunner (2011 a: 65-66).

14 Weiterführende Referenzen zu den unterschiedlichen Materialsorten und deren Methodologisierung siehe Brunner (2011 a: 57-79). 
(1999); für Buchumschläge und andere Illustrationen Regener (2006) und Breckner (2007); für Paratexte Genette (2001).

Am Ende dieses zweiten Analysedurchgangs konnte die Darstellungslogik der Ergebnisse festgelegt werden, die ich im Folgenden in drei Schritten als Variablen der Sinnformel präsentiere. ${ }^{15}$ Zuerst werden die Konturen des Wissensobjekts nachgezeichnet. Dabei geht es um die Verfolgung chronologischer Spuren durch das Material, Definitions- und Deutungspräferenzen sowie den Impetus der Bekämpfungsperspektive in Zusammenhang mit der Anwendungsorientiertheit der Terrorismusforschung. Eine Analyse von Buchumschlägen, die diese Forschung begleitet, ergänzt die Konturen, die sich im Verlauf von 20 Jahren herausgebildet haben. ${ }^{16}$ Darauf folgt eine Diskussion der Wege zum Wissen. Dabei wird gezeigt, wie bereits theoretische und methodische Zugänge dafür mitverantwortlich sind, welches Wissen auf welche Weise als Faktum präsentiert wird. Als bemerkenswerteste Ergebnisse werden in diesem Abschnitt die Verschiebung von Forschungsperspektiven vom Individuum über Organisationen hin zu Gesellschaften und ganzen Regionen, die Praxis qualitativer Interviews und ihre Schwächen, die Etablierung von Forschungsdatenbanken sowie die grafische Theoretisierung in Modellen diskutiert. ${ }^{17}$ Im dritten Schritt werden sechs Modi der okzidentalistischen Selbstvergewisserung dargestellt, die das Ergebnis insbesondere der Textanalysen darstellen. Es sind Pathologisierungen, (Ir-)Rationalisierungen, Sexualisierungen, Historisierungen, Geopolitisierungen und Kulturalisierungen, ${ }^{18}$ die sich für die spezifische Herausbildung des Wissensobjekts Selbstmordattentat als konstitutive diskursive Rahmungen sowie als sinnformelhafte Zuspitzungen der zunehmenden Vereindeutigung im Terrorismuswissen zeigen lassen.

\section{Variablen der Sinnformel I: Konturen des Wissensobjekts}

Auf Basis der chronologischen Darstellung von Ein- und Ausschlüssen, Verdichtungen, Kanonisierungen und Standardisierungen von unterschiedlichen (sozi-

15 Auf detaillierte Analysebeispiele wurde in diesem Aufsatz zugunsten einer Gesamtdarstellung der Ergebnisse im Überblick bewusst verzichtet, weshalb Verweise auf konkrete Textstellen im Folgenden nur kursorisch angeführt werden. In diesem Text gebe ich im Sinne der Argumentation eines Zusammenhangs von epistemischer und politischer Gewalt der Darstellung darauf basierender Analysen und Interpretation den Vorzug.

16 Ausführlicher dazu siehe Brunner (2011 a: 81-151). Für eine erweiterte und aktualisierte Argumentation und Durchführung einer wissenssoziologisch fundierten, postkolonial-feministisch verorteten Bildanalyse von Buchumschlägen am Beispiel des Wissensobjekts Selbstmordattentat siehe auch Brunner (2012a).

17 Ausführlicher dazu siehe Brunner (2011 a: 151-220).

18 Ausführlicher dazu siehe Brunner (2011 a: 220-340). 
al-)wissenschaftlichen Wissensbeständen werden nicht nur zeitliche und auf die Organisationslogiken des Feldes bezogene, sondern auch inhaltliche Konturen in der Herausbildung des Wissensobjekts Selbstmordattentat deutlich. Eines der für meine Fragestellung zentralen Ergebnisse ist die Verengung des definitorischen Spektrums, die sich im untersuchten Material über die zumeist implizite Ineinssetzung oder unkommentierte Verschiebung der Arbeitsbegriffe suicide bombing und suicide terrorism durchsetzt. Zwischen diesen beiden Begriffen steht eine Frage, die der gesamten Terrorismusforschung zugrunde liegt, die ihre Existenzberechtigung als sich herausbildende eigene Disziplin ausmacht und dennoch kaum explizit diskutiert wird: jene nach der Grenze zwischen legitimer und nicht legitimer, zwischen legitimierbarer und nicht legitimierbarer politischer Gewalt. ${ }^{19}$

Dieses Ergebnis steht in Zusammenhang mit einer bemerkenswerten Nicht-Thematisierung von staatlich oder international legitimierter militärischer Gewalt, gegen die sich Selbstmordattentate auch richten und von der sie bekämpft werden. Damit in Zusammenhang stehend ist wiederum die Dringlichkeit und Bekämpfungsperspektive zu verstehen, die sich in den untersuchten Wissensbeständen manifestiert. Sie hält das Terrorismuswissen über Selbstmordattentate jenseits disziplinärer, wissenspolitischer und weltanschaulicher Unterschiede zusammen.

Dass das Wissensobjekt Selbstmordattentat nicht nur für terrorismusforschende ExpertInnen und PolitikberaterInnen von Interesse ist, sondern vielfältige interdiskursive Anschlussstellen bietet, zeigt eine vergleichende Analyse von Buchumschlägen, die ich als Schnittstellen zwischen wissenschaftlichem Spezialwissen und außerwissenschaftlichen Wissensbeständen (vgl. Loew 2006) verstehe. An diesen »ideografischen " (McGee 1980) Verdichtungen wird auch jenseits einer textlichen Ebene sichtbar, worin die ideologisch aufgeladenen Momente der Vereindeutigung des Wissensobjekts und damit auch die Sinnformelhaftigkeit der »Konfliktverschärfung im Geiste einer Kulturkampfstimmung« (Brücher 2004: 7) bestehen. Beispiele für die visuelle Vereindeutigung des Terrorismuswissens sind etwa die Inszenierung des Sujets Sprengstoffgürtel, die spezifische Orientalisierung männlicher und weiblicher Figuren, deren visuelle Fragmentierung (bei Männern) bzw. Personifizierung (bei Frauen) oder die Betonung einer territorial gleichmäßig verteilten Globalität des Phänomens. Fast allen ist gemeinsam, dass sie einen Grad an Plakativität und Ideologisierung aufweisen, der für wissenschaftliche Bücher be-

19 Ich denke, dass diese Gratwanderung auch die Friedens- und Konfliktforschung seit ihren Anfängen beschäftigt, und dass es auch in diesem Feld keineswegs üblich ist, diese heikle Diskussion explizit zu führen. Eine eingehendere Beschäftigung mit dem Konzept der epistemischen Gewalt könnte diese Debatte voranbringen, indem sie Wissenschaft erneut als Teil von Herrschaftsverhältnissen problematisiert. 
merkenswert und zugleich gut an außerwissenschaftliches Wissen zum Thema anschlussfähig ist.

\section{Variablen der Sinnformel II: Wege zum Wissen}

Nicht nur die politischen und historischen Rahmenbedingungen der untersuchten Wissensproduktion und die darin verhandelten Inhalte verleihen dem Wissensobjekt Selbstmordattentat seine spezifische Verfasstheit. Es sind auch die Mittel und Wege, die (wissenschaftlichen) Methoden und ihre Anwendung, die für dessen spezifische Konturiertheit sorgen. So hat sich etwa die anfängliche Praxis des in individualpsychologischen Prämissen verwurzelten profiling der Terrorismusbekämpfung auch in der sozialwissenschaftlichen Forschung weit verzweigt. Die Vorstellung, dem Untersuchungsgegenstand und seinen primären AkteurInnen ein Gesicht zu geben und sie damit fassbar zu machen, äußert sich auf unterschiedlichen Ebenen. Sie reicht von der plakativen Illustration von Büchern über die Vorstellung eines generalisierbaren Adornoschen »autoritären Charakters« der Untersuchten bis hin zur Praxis der Erstellung von prototypischen Portraitierungen einzelner AkteurInnen auf Basis mehr oder weniger umfangreicher Informationssammlungen aus Massenmedien in Textform.

Angesichts offensichtlicher methodologischer Schwächen dieses Zugangs zur Wissensgenerierung hat sich im Anschluss daran die Praxis des Interviews als beliebte Methode durchgesetzt. Was an dieser »Feldforschung hinter Gittern« (Brunner 2011 a: 173) auffällt, ist die marginale Reflexion der Untersuchungssettings etwa in Gefängnissen bei gleichzeitiger Ausblendung der eigenen Position als WissenschaftlerIn. Die Art und Weise, wie aus in Interviews gewonnenem und geronnenem Wissen ein für die Forschung zu Selbstmordattentaten generalisierbares Wissen präsentiert wird, lässt beträchtliche Auslassungen und Vereindeutigungen erkennen, die - gestützt von einer Art Authentizitätsbonus der Interviewten sowie über die reklamierte Erfahrungsexpertise sowohl der Befragten als auch der Befragenden - Anspruch auf Exklusivität, Objektivität und vor allem Wahrheit vermitteln.

Als dritter Weg zum Wissen kann der zunehmende Rückgriff auf Datenbanken genannt werden, die im Laufe der Beschäftigung mit dem Gegenstand entstanden sind. Dabei zeigt sich, dass das darin gespeicherte Wissen aus je konkreten Fallbeispielen allmählich eine spezifische Generalisierung und auch infolgedessen eine weitreichende Distribution erfährt. Was dabei kaum thematisiert wird, sind die methodologischen Probleme, die in Zusammenhang mit ihrer Erstellung und Verwendung entstehen, sowie deren Einfluss auf die Ergebnisse von Untersuchungen. Be- 
merkenswert dabei ist insbesondere die Durchdringung dieser wissenschaftlichen Datenbanken mit medial generiertem, journalistischem Wissen, das auf diesem Wege einen oft ungerechtfertigten Validitätsgewinn erzielt.

Als methodisches Pendant zu diesem Befund ist die theoretisierende Modellbildung zu nennen, die sich vor allem in grafischen Verdichtungen niederschlägt. Dabei werden als zentral identifizierte Parameter und/oder zu zentralen Parametern gemachte theoretische Annahmen verdichtet und anschaulich zueinander in Beziehung gesetzt, wodurch spezifische Generalisierungen beschleunigt werden. Auch hier zeigt sich eine deutliche Abtrennung der untersuchten Gewalt von nationalstaatlich organisierten Gewaltformen sowie eine visuelle Symmetrierung des Forschungsgegenstandes, die die asymmetrischen Konstellationen von Gewalt-, Herrschafts- und Wissensverhältnissen aus den Verdichtungen des Wissens tilgt.

Als Ergebnis der Untersuchung unterschiedlicher Wege zum Terrorismuswissen kann festgehalten werden, dass eine allmähliche Verlagerung der Forschung von Attentat und AttentäterInnen über Organisationen und Gruppen hin zu Gesellschaften und Konfliktregionen stattgefunden hat. Parallel mit dieser Entwicklung verlaufend kann eine zunehmende Tendenz zur Kulturalisierung und Religiosisierung festgestellt werden, die eine anfängliche Psychologisierung mit Fokus auf die primären AkteurInnen sowie eine sich davon abgrenzende Rationalisierung unter der Betonung organisations- und gruppenbezogener politischer Interessen allmählich abgelöst hat. Dementsprechend verschieben sich auch die Untersuchungseinheiten vom Individuum über die Gruppe bis hin zu Konzeptionen des Gesellschaftlichen - ohne jedoch die Prämissen der Anfänge (Pathologisierungen, Psychologisierungen) hinter sich zu lassen. Im Gegenteil, diese werden gewissermaßen modifiziert, um den sich vergrößernden Untersuchungseinheiten angepasst zu werden. Die Generalisierung kulturalistischer und religiosisierter Erklärungen auf dieser Ebene begünstigt wiederum deren Verfestigung im Sinne einer okzidentalistischen Selbstvergewisserung, die über jeweils einzelne Untersuchungsgegenstände und Phänomene hinausgeht und zu einem paradigmatischen Zugang zu politischen und sozialen Entwicklungen wird.

In diesem Zusammenhang ist schließlich ein weiteres wichtiges Ergebnis zu nennen. Obwohl die Forschung insgesamt immer wieder komparatistisch nach Fallbeispielen sucht und diese etwa in den Konflikten von Tamil Eelam/Sri Lanka, Tschetschenien/Russland oder Kurdistan/Türkei findet, hat sich eine auffällige Dynamik des pars pro toto durchgesetzt, den das palästinensisch-israelische Beispiel in der Forschung insgesamt einnimmt. Die immer wieder an diesem Beispiel festgemachte Vorstellung einer primär islamistischen Bedrohung, die mit vielfältigen Orientalisierungen einhergeht, hat allerdings weniger mit der Quantität oder Qualität dieser 
konkreten Anschläge zu tun als vielmehr mit den ein- und ausschließenden Logiken und Organisationsstrukturen der Herausbildung von vermeintlich eindeutigem Wissen darüber, was ein Selbstmordattentat ist.

\section{Variablen der Sinnformel III: Modi der okzidentalistischen Selbstvergewisserung}

Die Analyse des Materials lässt sechs Modi der okzidentalistischen Selbstvergewisserung benennen. Diese verlaufen zwar nicht linear und statisch, weisen aber einige bemerkenswerte überlappende bzw. komplementäre Merkmale auf. In ihnen treten die Prozesshaftigkeit und Dynamik des Wissensobjekts sowie die Tendenz zu bestimmten Vereindeutigungen, Auslassungen und Ambivalenzen seiner Hervorbringung zutage. Entlang dieser spezifischen Herstellungen von Andersheit wird deutlich, welche Vorstellungen von Eigen-heit und Normalität von politischer Gewalt sowie von einer zunehmend kulturalisierten, rassisch nicht markierten, vermeintlich ausschließlich säkularen, politisch und vor allem moralisch überlegenen Westlichkeit für das Wissensobjekt und die Sinnformel Selbstmordattentat konstitutiv sind. Mit der dichotomisierten Herstellung von Andersheit und Eigen-heit im und über das Wissensobjekt wird nämlich nicht nur die Dominanz eines westlichen self in power im Sinne einer ökonomischen, militärischen und politischen Vormachtstellung befestigt, sondern auch dessen symbolisch-kulturelle Dimension. Die im Prozess einer okzidentalistischen Selbstvergewisserung hervorgebrachten und befestigten Eigenheiten werden dann zu einer ebenso essenzialisierbaren Eigen-heit, die in einem positivistischen Verständnis kaum mehr hinterfragbar erscheint. Die jeweils spezifisch gewordenen Konturen des Wissensobjekts gerinnen damit zur universalisierten Vereindeutigung durchaus heterogener und vielschichtiger Gewalthandlungen, die eine zunehmende Sinnformelhaftigkeit und Selbstverständlichkeit erlangt hat.

Vor diesem Hintergrund können die »geteilten Geschichten« (Randeria 1999) der Terrorismusforschung und ihrer Gegenstände neu bewertet und damit auch politische Macht-, Gewalt- und Herrschaftsverhältnisse in der Gegenwart relationaler kontextualisiert werden. Die hier dargelegten Analysen und Interpretationen stellen insofern keineswegs eine Verharmlosung realer physischer Gewalt in Form von Selbstmordattentaten dar und dienen auch nicht deren Rechtfertigung. Im Gegenteil - mit der Benennung der Dimension von epistemischer Gewalt und ihrer Verwobenheit mit physischen und strukturellen Gewaltformen und -verhältnissen kann ein vollständigeres, den komplexen Realitäten von Vergangenheit, Gegenwart und Zukunft angemesseneres Bild dessen entstehen, wovon noch lange zu sprechen sein 
wird: Legitimität und Illegitimität, Hintergründe und Konsequenzen von politischer Gewalt - in der Vielfalt und Verwobenheit ihrer Erscheinungsformen und Zusammenhänge. Wissenschaft ist darin tief verstrickt. Auch oder sogar insbesondere die Friedens- und Konfliktforschung ist aufgefordert, diesem Umstand Rechnung zu tragen, will sie die globalen Rahmenbedingungen nicht aus dem Blick verlieren, innerhalb derer Frieden und Konflikte stattfinden.

Im Folgenden stelle ich die sechs von mir identifizierten Modi vor, die in der Verwobenheit ihrer Erscheinungsformen und in der vermeintlichen Natürlichkeit ihrer Effekte die okzidentalistische Selbstvergewisserung im hegemonialen Terrorismuswissen gewährleisten. Zur Illustration des Gesagten werden eingangs ausgewählte Zitate aus dem Material angeführt, die im Kontext der einzelnen Kapitel repräsentativ sind.

\subsection{Pathologisierungen}

»The position of the student of terrorism is not unlike that of a physician dealing with a disease, the exact causes of which remain unknown to this day, or a drug of which it is not known how precisely it functions. But this will not prevent him from diagnosing the disease, or from prescribing the drugs that are applicable« (Laqueur 2003: 238).

Trotz aus sozialwissenschaftlicher Perspektive heute offenkundiger Probleme und Widersprüche finden sich Pathologisierungen an vielen Stellen im Terrorismuswissen. ${ }^{20}$ Mit ihnen gehen Vorstellungen von umfassend bedrohter Gesundheit (der "westlichen« Welt), von der (Un-)Möglichkeit der Heilung der Andersheit (der "Anderen «) und insbesondere von möglichen Immunisierungsmaßnahmen gegen das als krankhaft Definierte einher. Damit sind oft nicht nur einzelne Personen oder Gruppen gemeint, sondern eine generalisierte Andersheit, die in der Sinnformel Selbstmordattentat eine ihrer am schärfsten konturierten Ausprägungen findet. Zugleich wird in diesem Modus auch eine Vorstellung psychischer und mentaler Gesundheit gestärkt, mit der die aus dieser Perspektive sprechenden DiskursakteurInnen selbst sowie die ihnen zugeordneten geografischen und politischen Räume belegt werden. Dabei wird auch die Doppelrolle der Forschenden als zumindest potenzielle DiagnostikerInnen und HeilerInnen befestigt. Bilder der Pathologisierung haben sich aus den Anfängen der auf das Individuum fokussierten psychologischen

20 Die Zusammenfassung bezieht sich insbesondere auf Analysen aus Sprinzak (2000), Laqueur (2003), Davis (2003), Stern (2003), Reuter (2004), Victor (2004), Bloom (2005), Schweitzer (2006) und Berko (2007). 
und kriminologischen Forschung zum Thema gebildet und sind im Laufe der Jahre durchaus von komplexeren Konzeptionen kritisiert und auch verdrängt worden. Sie sind aus dem überwiegend positivistischen Terrorismuswissen jedoch bis heute nicht gänzlich verschwunden. Ganz im Gegenteil haben sich pathologisierende Begriffe und Annahmen mit der Ausweitung des Forschungsfokus verbreitet und sind von Teilen sozialwissenschaftlicher Perspektivierungen übernommen worden. Das heißt zugleich, dass zunehmend nicht nur mehr die primären AkteurInnen von Selbstmordattentaten pathologisiert werden, sondern mitunter ganze Bevölkerungsgruppen oder Weltregionen, die als kollektiv deviant/krank oder eben auch normal/ gesund verstanden werden.

\section{2. (Ir-)Rationalisierungen}

»Perhaps, the main difference is that SM [suicide mission; Anm. d. A.] goals are often less of decisive transparent importance than those of war heroes. The hero is quintessentially an instrumental altruist, while the suicide bomber may not always know very well what he is dying for « (Gambetta 2005: 278).

Eine Form der chronologischen Weiterentwicklung des Modus der Pathologisierungen ist die Verlagerung auf eine Diskussion von Rationalität und Irrationalität von TäterInnen und Tat. ${ }^{21}$ Diese geht oft Hand in Hand mit einer Kontrastierung von illegitimierter terroristischer versus legitimierter staatlich organisierter Gewalt. Die schmale Grenze zwischen bedrohlichen MärtyrerInnen aus einem diffus generalisierten Orient einerseits und im Westen verorteten Kriegshelden andererseits ist im Modus der changierenden (Ir-)Rationalisierungen hart umkämpft. Auch dieser Modus hat sich vom Individuum über die Organisationen hin zum Bereich des Gesellschaftlichen verschoben. Eine darüber hinausreichende weitere Ebene - etwa staatlicher oder zwischenstaatlicher Gewaltformen in der Konstellation globaler asymmetrischer Herrschaftsverhältnisse - bleibt in diesem Modus weitgehend unbenannt. Nicht mehr Gesundheit und Krankheit erklären die unterstellte Disposition zu extremer Gewalt auf Seiten von SelbstmordattentäterInnen, sondern die vorhandene oder fehlende Vernunftfähigkeit. Die partielle Rationalisierung des Forschungsgegenstandes, seiner AkteurInnen und Rahmenbedingungen bildet zwar eine gewisse Abgrenzung gegenüber früheren psychologisierenden und pathologisierenden Ansätzen, aber nur in begrenztem Maße gegenüber deren zunehmender Kulturalisierung. Diese ist nämlich mit den unterschiedlich verwendeten Parame-

21 Die Zusammenfassung bezieht sich insbesondere auf Analysen aus Sprinzak (2000), Elster (2005), Gambetta (2005) und Holmes (2005). 
tern der Rationalisierbarkeit politischer Gewalt durchaus kompatibel und unterstützt diese zum Teil sogar. An dieser Stelle kommen auch TrägerInnen staatlicher Gewalt zum diskursiven Einsatz: Helden und Soldaten. Spätestens hier wird dann auf das anfänglich oft zitierte, weil qualitativ und quantitativ herausragende, aber zunehmend schwieriger mit dem Wissensobjekt Selbstmordattentat kompatible Beispiel der japanischen Kamikaze als reguläre Soldaten im Zweiten Weltkrieg verwiesen, das in den Anfängen der Forschung zum Thema noch als Vergleichsbeispiel par excellence herangezogen wurde. An seinem allmählichen Verblassen wird deutlich, dass die Frage der Rationalisierbarkeit von politischer Gewalt eng verwoben ist mit jener nach deren prinzipieller Legitimierbarkeit im Rahmen nationalstaatlicher Armeen. So genannte Selbstmordkommandos im Rahmen nationalstaatlich legitimierter Gewalt (wie die japanischen Kamikaze) werden als Vergleichsfolie für ein gegenwärtiges Wissensobjekt daher zunehmend unplausibler und in Bezug auf die Konturierung des Wissensobjekts Selbstmordattentat irrelevanter. Immer in Mode scheint jedoch die Vergeschlechtlichung und Sexualisierung von politischer Gewalt zu sein, die der feministischen Forschung seit langem bekannt ist und sich in spezifischer Weise auch im Terrorismuswissen niederschlägt.

\subsection{Sexualisierungen}

") You've come a long way, baby!< Virginia Slim cigarette ads used to read. After years of struggle, the women's movement in the $1970 \mathrm{~s}$ brought men and women in the first world to a level of relative parity in most areas of employment, status, and opportunities. However, in the rest of the world, the position of women remains seriously disadvantaged compared to that of men. According to a well cited UNDP report, women in Africa and in the Middle East suffer most from inequality. In one area, however, women in the developing world seem to be making their mark in achieving parity with men - in perpetrating suicide terrorism« (Bloom 2005: 142).

Dreh- und Angelpunkt des Modus der Sexualisierungen ist die nachhaltig orientalisierte Vorstellung eines dislozierten paternalistischen Patriarchats, das in verschiedenen Ausprägungen sowohl für von Männern als auch für von Frauen verübte Anschläge als relevanter Erklärungsfaktor etabliert wird. Demgegenüber wird der Raum der eigenen SprecherInnenposition insbesondere in Geschlechterfragen als emanzipiert und fortschrittlich inszeniert. ${ }^{22}$ Orientalisierte Männlichkeit und eine

22 Die Zusammenfassung bezieht sich insbesondere auf Analysen aus Israeli (1997), Juergensmeyer (2003), Laqueur (2003), Victor (2004), Bloom (2005), Skaine (2006). 
daran geknüpfte terroristische Gewaltneigung mit sexualisierten Elementen spielen dabei ebenso eine Rolle wie die Idee, dass Selbstmordattentäterinnen entweder von männlichen Angehörigen zur Tat genötigt oder aber für eine missverstandene Frauenbefreiung kämpfen würden. An diesem Modus ist die Durchlässigkeit zu journalistischen Wissensbeständen besonders auffällig. Die Tatsache, dass sich sexualisierte Stereotypisierungen besonders hartnäckig auch im wissenschaftlichen Spezialwissen verfestigen, steht auch mit dieser Durchlässigkeit in einem Zusammenhang. Konkret sind es an Bildern und Metaphern reiche Geschichten über offensichtlich auch für WissenschaftlerInnen unwiderstehliche, im Jenseits auf einen islamistischen Märtyrer wartende Jungfrauen ${ }^{23}$, der Verweis auf die immer wieder in ihrer Rolle als Frauen positionierten »MärtyrerInnenmütter« oder der Begriff der »Schwarzen Witwen«, der sich für tschetschenische Attentäterinnen durchgesetzt hat. All diesen Frauenfiguren gemeinsam ist das darin vermutete Erklärungspotenzial über eine vorrangig im Privaten und Sexuellen verortete Gewalt- und Handlungsfähigkeit von Frauen, die deren politische Gründe und Ziele deutlich schwächt. Die immer wiederkehrende Rezeption dieser Figuren und der zumeist journalistischen Berichte, aus denen sie stammen, zeigt wenig Interesse an Fragen der Validität eines von der (bislang der Geschlechterdemokratie gänzlich unverdächtigen) Terrorismusforschung entdeckten Genderwissens, das diesen Namen kaum verdient. In Hinblick auf feministische Fragestellungen und Theoriebildung ist von Bedeutung, dass eine Art »embedded feminism « (Hunt 2006) sowohl im wissenschaftlichen als auch im außerwissenschaftlichen Terrorismuswissen fest verankert und für dessen Okzidentalismus konstitutiv ist. ${ }^{24}$ Die damit gemeinte Indienstnahme feministischer Motive und Diskurse sowie die Fokussierung speziell auf Selbstmordattentäterinnen und deren angeblich spezifisch weibliche Motive dienen selten der Klärung der jeweils konkreten Umstände. Beinahe immer hingegen nützen sie der um einen diffus konturierten und großzügig verwendeten »Faktor Gender« angereicherten Verteidigung so genannter westlicher Werte im Namen von Frauenbefreiung und Geschlechtergerechtigkeit (vgl. Brunner 2007; Dietze 2009). Eine bemerkenswerte neuere Entwicklung ist nicht weniger problematisch, nämlich die Rebiologisierung von politischer Gewalt, die über eine ebenso verkürzte wie problematische Genderperspektive biologische Rassismen wieder salonfähig zu machen scheint. Was im gesamten Untersuchungszeitraum (1996-2007) zwar punktuell vorhanden war, aber kaum rezipiert wurde, hat mit einem Aufsatz von 2010

23 Im Rahmen eines späteren Aufsatzes bin ich der Frage nach der diskursiven Prominenz der 72 Jungfrauen und deren Reiz für die Terrorismusforschung nachgegangen (Brunner 2012 b).

24 Siehe dazu auch Brunner (2011 b), ein Aufsatz, in dem ich diese Dynamik ausführlicher diskutiere und aus geschlechtertheoretischer Perspektive weiterdenke. 
wieder Eingang in das Feld des kanonisierten Terrorismuswissens gefunden. In diesem wird von zwei PolitologInnen (Thayer/Hudson 2010) ein expliziter Zusammenhang zwischen spezifisch islamischem Selbstmordterrorismus und Evolutionsbiologie hergestellt. Männliche Sexualität, der Kampf um Reproduktionschancen und als vorzivilisiert gerahmte Geschlechterverhältnisse in als isoliert verstandenen islamischen Gesellschaften werden dabei zum primären Faktor für eine angenommene biologische Prädisposition zum Selbstmordterrorismus erklärt.

\subsection{Historisierungen}

»Suicide terror can be found as early as the tenth century in the Hashashin (Assassins) sect. From the beginning of the eighteenth century, the pattern of suicide attacks was adopted by Moslems waging their battle against Western colonialism in Asia. However, the >modern ‘ manifestations of the suicide terror phenomenon surfaced with the appearance of the first suicide terrorists in Lebanon about twenty years ago« (Shay 2004: 1).

Selbstmordattentate werden von der Terrorismusforschung oft als radikal neues, historisch einzigartiges und den so genannten Neuen Terrorismus besonders eindrücklich verkörperndes Phänomen bezeichnet. Nichtsdestotrotz nehmen die meisten ForscherInnen eine Historisierung des Untersuchungsgegenstandes vor, die jedoch vorrangig der Rahmung der Forschungsperspektive dient und keiner umfassenden geschichtswissenschaftlichen Aufbereitung der jeweiligen Zugänge zum Thema gerecht werden kann. In diesem Punkt der expliziten und impliziten (Nicht-)Historisierung zeigt sich in der Hervorbringung des Wissensobjekts ein generelles Merkmal der Terrorismusforschung, auf das Isabelle Duyvesteyn (2004) kritisch hinweist. Als vierten Modus habe ich dementsprechend jenen der Historisierungen herausgearbeitet und auffallend kontrastierende Bezugnahmen auf Selbstmordattentate im Zweiten Weltkrieg, im antikolonialen Widerstand in Südostasien vom 16. bis zum 18. Jahrhundert sowie auf die oft als Sekte bezeichnete Gruppe der Assassinen aus dem 11. Jahrhundert diskutiert. ${ }^{25}$ Bemerkenswert ist dabei insbesondere, dass die jüngste Geschichte der jeweiligen politischen Konflikte, in denen Selbstmordattentate verübt werden, die politische Situation der langen Jahrzehnte des so genannten Kalten Krieges sowie Querverbindungen zu aktuellen geopolitischen Entwicklungen insgesamt von den Historisierungen der jeweiligen Fallbeispiele abgeschnitten werden (vgl. Mamdani 2005). Zum privilegierten

25 Die Zusammenfassung bezieht sich insbesondere auf Analysen aus O’Neill (1981), Dale (1988), Kushner (1996), Israeli (1997), Sprinzak (2000), Reuter (2004) und Shay (2004). 
wie isolierten Dreh- und Angelpunkt der jüngeren Geschichte werden hingegen die Anschläge im Libanon Mitte der 1980er Jahre gemacht. An diesen bestens anschlussfähig ist die auffällige Präsenz der Referenz auf die so genannten Assassinen im heutigen Iran und Syrien, die mit heutigen AttentäterInnen bestenfalls ihre stereotypisierten und ideologisierten Fremdzuschreibungen teilen, weniger hingegen die Taktiken und Ziele ihrer als gezielte Tötungen zu bezeichnenden Anschläge. Beides wiederum ist mit der impliziten und expliziten Privilegierung des palästinensischen Beispiels kompatibel, das nicht nur aufgrund der dazu äußerst umfangreichen Forschungen verstärkt generalisierend für das Wissensobjekt wirkt. Historische Kontinuitäten werden nämlich nicht vorrangig über Mittel und Ziele der untersuchten Gewaltformen hergestellt, sondern über die Verortung des Phänomens in einer kulturalisierten und religiosisierten Sphäre einer kollektiviert skizzierten arabischen Welt, die sich insbesondere über identitäre Homogenität und Kontinuität auszeichne. Entlang derselben Kategorisierungen - Kultur und (Nicht-)Religion treten auch die Konturen eines hierarchisch übergeordneten Okzidents immer deutlicher hervor. In diesem verortet sich die Forschung selbst, von diesem aus spricht sie und entwirft als Terrorismusbekämpfungsforschung auch politikberatende Handlungsempfehlungen.

\subsection{Geopolitisierungen}

»If not for the world's interest in Persian Gulf oil, the obvious solution - just as it was for Ronald Reagan when the United States faced suicide terrorism in Lebanon - might well simply be to abandon the region altogether. This, however, is not possible, certainly not for the foreseeable future. Thus the question is: can we find a lasting solution to suicide terrorism that does not compromise our core interest in maintaining access to one of the world's key oil-producing regions?« (Pape 2005: 237).

Im Modus der Geopolitisierungen des Wissensobjekts kommen nicht nur dessen behauptete ganz andere Andersheit, sondern vor allem die sich darüber mit konstituierenden Eigen-heiten des Terrorismuswissens selbst sowie die vermeintliche Abgetrenntheit des einen vom anderen in den Blick. Hier verdichten sich die vielschichtigen Dimensionen epistemischer Gewalt, die das Wissen über und die Bekämpfung von Terrorismus sowie die Wandelbarkeit der Legitimierbarkeit von politischer Gewalt prägen. ${ }^{26}$ Das Wissensobjekt Selbstmordattentat scheint eine be-

26 Die Zusammenfassung bezieht sich insbesondere auf Analysen aus Israeli (2003), Atran (2003; 2006), Reuter 2004), Khosrokhavar (2005), Pape (2005) und Lewis (2007). 
deutsame Funktion darin zu haben, räumliche Distanzen auf dem Globus und in den Köpfen zu schaffen und damit auch geopolitische Räume und deren Lebensrealitäten voneinander getrennt zu halten. Im spezifischen Verbinden und Trennen, in den spezifischen Nähen und Distanzen, die sich im Terrorismuswissen herausbilden, liegt ein zentrales Moment epistemischer Gewalt, auf das meine Argumentation abschließend zugeht. Das Augenmerk auf geopolitischen Raum zu legen ermöglicht, das Wissensobjekt und die Möglichkeitsbedingungen seiner Hervorbringung wieder in einen Zusammenhang mit der Ausgangsfrage nach epistemischen Gewaltverhältnissen zu stellen und mit den Standorten von Diskurspositionen und WissensakteurInnen zusammenzudenken. In diesem Modus zeigt sich, auf welche Weise Standorte und Standpunkte hegemonialer Terrorismusforschung und -bekämpfung miteinander verwoben sind. Schließlich bin ich anhand des untersuchten Materials zur Frage der Unterscheidbarkeit zwischen dem Eigenen und dem Anderen zurückgekommen, die im Material mit einer Entgrenzung der Bekämpfungspraxis ebenso wie mit einer möglichen Zunahme von Selbstmordattentaten auch jenseits bislang bekannter AkteurInnen, Konflikte und Ziele zusammengedacht wird. Entgegen und gerade auch aufgrund der im Wissensobjekt sichtbaren Vereindeutigungen, so meine These, besteht die spezifische Konturierung des Untersuchungsgegenstandes nicht nur in dessen vermeintlicher Andersheit, sondern auch in einer okzidentalistischen Abwehr der Annahme möglicher Ähnlichkeiten zwischen illegitimer und legitimer Gewalt, zwischen Selbstmordattentaten und anderen Mitteln der Konfliktaustragung. In die Trennung des Einen und des Anderen sowie des Eigenen vom Anderen, die nie nur eine analytische, sondern immer auch eine politische ist, wird von der Terrorismusforschung viel Energie investiert; und nicht alles, was gewusst werden kann, fließt gleichermaßen in hegemoniale Wissensbestände ein. In diesem Modus wird sehr deutlich, dass die Terrorismusforschung nicht lediglich politische Gewalt verstehen und erklären will. Viele der terrorismusforschenden »intellectuals of statescraft« (Ó Tuathail 1996) suchen auch nach guten Gründen dafür, sich selbst und den geopolitischen Raum ihrer eigenen Verortung und Zugehörigkeit im Verhältnis zur von ihr analysierten Gewalt zu positionieren, ohne mit deren Entstehungsbedingungen in Verbindung gebracht zu werden. Dies geschieht mitunter mit einem klaren Bekenntnis zu geopolitischen Interessen, die jenseits der Terrorismusbekämpfung selbst liegen, wie obiges Zitat deutlich zeigt.

\subsection{Kulturalisierungen}

»We are talking, in other words, about two worlds apart, separated by an unbridgeable cultural gap: the one, modern, open, tolerant, advanced, well- 
meaning, law-abiding, democratic, orderly, eager to live and let live, oriented toward progress and the future, accepting and self-confident to the point of running the risk of self-destruction by generously allowing into itself Muslim elements bent on terrorizing it; the other, narrow-minded, bigoted, jealous, backward, lawless, bent on restoring past glory, intolerant of others and other ideas, tyrannical in rule, unable to accept and include, suspicious and paranoid, taking shadows of things as things themselves, vengeful and vindictive, prone to humiliation and shunning exposure and shame, and ready to waive its own life and to take down with its Western enemy« (Israeli 2003: 417).

Die aus sozialwissenschaftlicher wie aus friedensforschender Perspektive beunruhigendste Entwicklung des hier untersuchten Wissensobjekts ist die zunehmende Kulturalisierung und Religiosisierung von sozialen und politischen Ereignissen und Verhältnissen. Diese betrifft nicht nur die Erklärungsweisen der jeweiligen Untersuchungsgegenstände, sondern auch die ihnen zugrunde gelegten Begriffe, Konzepte und Theoretisierungen. In diesem Prozess erleben eurozentrische Vorstellungen von einem kohärenten, restlos aufgeklärten, vorbildlich demokratischen und damit per se mit globalen Dominanzansprüchen ausgestatteten Abendland eine Renaissance, deren Effekte weit über die Hervorbringung einzelner unter diesen Prämissen hervorgebrachter Wissensobjekte hinausgehen. Die Rationalisierbarkeit und vor allem Legitimierbarkeit politischer Gewalt stößt dort an ihre Grenzen, wo auch eine generalisierte Andersheit jenseits des Individuums mit entworfen wird. Dies ist im Modus der Kulturalisierungen am eindrücklichsten erkennbar, in seiner Komplexität und Diffusität aber auch am schwierigsten nachzuweisen. ${ }^{27}$ Im Material kommt an vielen Stellen ein Kulturbegriff zum Tragen, der von deutlich voneinander abgegrenzten Räumen gekennzeichnet ist, die sich insbesondere entlang der Kriterien geopolitischer Verortung, religiöser Identität und damit oft verschränkten ethnisierten/rassifizierten Merkmalen der Andersheit manifestieren. Es ist ein solcher »Culture Talk« (Mamdani 2005), der es erlaubt, Moderne und politische Gewalt in einem spezifisch eurozentrischen Verhältnis zueinander zu denken, das geopolitische Räume als voneinander getrennte und Kulturen als in sich geschlossene Einheiten versteht. Wendy Brown bringt dieses problematische Verständnis von Kultur auf den Punkt. Die von Mahmood Mamdani problematisierte ideologische Kulturalisierung von Gewalt und Politik reduziert Konflikte und Differenzen nämlich nicht gleichermaßen auf das Kulturelle, so Brown (2006: 150). Vielmehr hält sie durchaus unterschiedliche Schattierungen davon bereit, die eine wirkmächtige

27 Die Zusammenfassung bezieht sich insbesondere auf Analysen aus Shay (2004), Pedahzur (2005), Ricolfi (2005) und Hafez (2006; 2007). 
Trennung des konservativen wie des liberalen Mainstreams immer wieder von Neuem befestigen: ») $[\mathrm{w}] \mathrm{e}<$ have culture, but culture has $>$ them , or we have culture while they are a culture « (Brown 2006: 151; Hervorh. im Orig.).

Neben der im vorigen Punkt angesprochenen Notwendigkeit einer Selbstreflexion in der Friedens- und Konfliktforschung hinsichtlich ihrer eigenen Geopolitisierungen ist der Modus der Kulturalisierungen jener, der für eine Weiterarbeit am Begriff der epistemischen Gewalt am dringlichsten erscheint. An ihm besteht meines Erachtens der größte Bedarf einer empirischen wie auch einer theoretischen Weiterentwicklung, denn »Kultur« oder »Kulturen« müssen nicht notwendigerweise als vorrangiger Erklärungsrahmen bemüht werden, wenn es darum geht, Gründe für die Anwendung nicht-staatlicher politischer Gewalt zu finden. Dasselbe gilt für die oft mit Kultur gleichgesetzte Kategorie des Religiösen. Gerade an diesem Punkt der Kulturalisierung und Religiosisierung des Phänomens wird deutlich, dass diese nicht nur der vermeintlichen Erklärung von Devianz des Anderen dient, sondern vor allem der Befestigung der Normalität des Eigenen. Diese Normalität an einen diffusen Begriff des (Anders-)Kulturellen und des (Nicht-)Religiösen zu koppeln, erweist sich in Bezug auf die Generalisierung des Wissensobjekts und dessen Vereindeutigung als Sinnformel als weitaus wirksamer und vermeintlich plausibler, als diese Eigen-heiten etwa mit politischen und historischen Fakten und in ihren relationalen Verbindungen mit den beständig hergestellten Andersheiten zu erklären. Die Tendenz, dass sozialwissenschaftliches Wissen sich in einem seiner gegenwärtig dynamischsten und quer über viele Disziplinen expandierenden Felder verstärkt einem Paradigma des diffus Kulturellen und Religiösen verschreibt, das explizit und implizit von sozialen, politischen, ökonomischen, militärischen und geopolitischen Kontexten der Vergangenheit und der Gegenwart abgetrennt wird, sollte kritische SozialwissenschaftlerInnen durchaus beunruhigen. Die hier im Überblick dargelegte Perspektive, die für die weitergehende Analyse von Zusammenhängen zwischen politischer Gewalt einerseits und epistemischer Gewalt andererseits plädiert, will ein Beitrag zu einer solchen - produktiven - Beunruhigung sein.

\section{Zusammenfassung und Ausblick}

Die wissenssoziologisch-diskursforschende Frage nach hegemonialen Wissensbeständen über ein auch diskursiv umkämpftes Thema fokussiert entsprechend ihrer Grundlegung auf jene Formen von Gewalt, die mit dem Wissen selbst zu tun haben. Dieses war Gegenstand der hier zusammengefassten Untersuchung. Auf der Ebene der Analyse von epistemischer Gewalt - und nicht etwa auf der Erklärung einzelner oder generalisierter Attentate - liegt dementsprechend der wesentliche Gewinn in 
Hinblick auf die Ergebnisse. Hegemoniale Terrorismusforschung versteht das von ihr produzierte Wissen als Ressource zur möglichen politischen Lösung des beforschten Problems, nicht jedoch als dessen Teil. In dieses Verhältnis und Verständnis interveniert die in diesem Aufsatz und in dem ihm zugrunde liegenden Buch dargelegte Perspektive. Ich habe darin problematisiert, inwiefern hegemoniales Terrorismuswissen zum Thema Selbstmordattentat selbst von epistemischer Gewalt durchzogen ist, die Macht-, Gewalt- und Herrschaftsverhältnisse weder kausal noch linear, aber doch prinzipiell mit konturiert. Es wurde diskutiert, in welchem $\mathrm{Zu}-$ sammenhang die diskursive Herstellung eines "ganz anderen Anderen « (Sarasin 2003: 49) im Wissensobjekt Selbstmordattentat mit konstitutiven Konzeptionen des Eigenen steht; das heißt in diesem Falle, eines staatlich und zwischenstaatlich organisierten Gewaltmonopols und dessen Verschränkung mit Wissen(schaft-)sproduktion in ihrer hegemonialen und imperialen Verfasstheit. Die Dominanz des Selbstverständlichen, weil Hegemonialen, kann nämlich erst dann erschüttert werden, wenn das Andere und die Andersheit im Zentrum eben dieses Subjekts (Yeğenoğlu 1999: 6), also in den epistem(olog)ischen, theoretischen und methodologischen Prämissen des hier untersuchten Spezialwissens selbst, verortet werden, wenn also entsprechend der hier argumentierten Denkbewegung das Moment der Gewalt als epistemische Dimension ins Innere der Terrorismusforschung selbst verlagert und dort in einem konstitutiven Zusammenhang mit politischer Gewalt und der Stabilisierung asymmetrischer Ungleichheitsverhältnisse gedacht wird. Genau das zu verhindern, gelingt dem Mainstream der Terrorismusforschung selbst an ihren kritischen Rändern gut. In einem gegenläufigen Sinne habe ich mit der Analyse eben dieser Forschung versucht, die dominante Struktur ihrer Selbstverständlichkeit zu irritieren und herauszufordern. Dazu erforderlich ist ein weiter und Kontroversen in Kauf nehmender Gewaltbegriff, der in feministischer und postkolonialer Theorie weitgehend konsensfähig und auch in der Friedensforschung und Teilen der Konflikt- und Gewaltforschung durchaus intelligibel ist. Einer Terrorismusforschung im engeren Sinne läuft ein weiter Gewaltbegriff hingegen grundsätzlich zuwider, stellt er doch den Forschungsgegenstand selbst und damit die sich neu herausbildende Subdisziplin per se in Frage.

Die liberale Annahme, dass das Feld der Politik, und erst recht das der Wissenschaft, strikt getrennt sei vom Feld der Gewalt, und dass es die vorrangige Aufgabe des modernen Nationalstaates und seiner Wissenschaften sei, Gewalt aus dem Feld der Politik auszuschließen und in den Bereich des Krieges zu verlagern, ist ebenso als Illusion wie als Technik der Macht im Sinne einer Ausprägung auch von epistemischer Gewalt zu verstehen. In einem solchen Verständnis wird am prinzipiell legitimierbaren Krieg der Einen dann lediglich der Exzess kritisiert, wohingegen 
am prinzipiell zu delegitimierenden Terrorismus der Anderen eine Essenz festgemacht wird (Asad 2007: 16-17). Dies geschieht in diskursiven Räumen der Hervorbringung, Aushandlung, Verwerfung und Verfestigung von Wissensbeständen, in die das hier untersuchte wissenschaftliche Spezialwissen eingebettet ist. Das darin hervorgebrachte Wissensobjekt Selbstmordattentat fungiert als sinnformelhafte Verdichtung dieser angenommenen und zugleich diskursiv hervorgebrachten Essenz. Es ist dementsprechend von spezifischen Gewissheiten durchzogen, die kritisch zu hinterfragen nicht nur eine wissenssoziologisch spannende Fragestellung, sondern auch eine politische Gratwanderung innerhalb wissenschaftlicher Debatten darstellt. Ein solches Fokussieren auf epistemische Gewaltasymmetrien habe ich nicht zuletzt deshalb unternommen, um »einen Blick auf den Zusammenhang zwischen Beobachteten und Beobachtenden zu ermöglichen, zwischen den Produkten und der Produktion, zwischen dem Wissen und dem Ort seiner Entstehung « (Coronil 2002: 184) - zwischen den und dem scheinbar so ganz anderen Anderen und dem Terrorismuswissen, das in der Position ist, dieses zu definieren, zu klassifizieren und zu analysieren.

Und was bedeutet dies jenseits des hier diskutierten Gegenstandes für die Friedens- und Konfliktforschung - zumal für jene, die sich keineswegs als anwendungsorientierte Gewaltforschung oder sogar als kritische Intervention in dieses Feld versteht? Eine friedenswissenschaftliche Diskussion des Zusammenhangs von politischer und epistemischer Gewalt muss intensiver als bislang darüber nachdenken, welche weit reichende Formen struktureller, symbolischer und auch direkter physischer Gewalt durch ihren eigenen Beitrag legitimierbar bleiben und werden. Dies hat auch mit der institutionellen, thematischen und schließlich auch mit der politischen Verortung in diesem Feld zu tun sowie mit dessen Ressourcen, Theorietraditionen, Methoden und Paradigmen, die in die zutiefst asymmetrische Organisation von Wissen und Macht eingelassen und von diesen nicht zu trennen sind. Gerade die Friedens- und Konfliktforschung ist immer wieder herausgefordert, ihre Positionierungen in Hinblick auf die globalen Rahmenbedingungen von Frieden und Konflikten zu hinterfragen und neu auszurichten. Dass dies kein konsensualer und kollektiver Prozess sein kann, liegt angesichts der unterschiedlichen Verortungen und Machtgefälle auch innerhalb dieses Feldes auf der Hand. Man kann nun einwenden, dass dies keineswegs eine neue Erkenntnis sei, und dass sich vor allem feministische Wissenschaft insgesamt durch Kritik am Wissenschaftsbetrieb auszeichnet, die sie zugleich als Herrschaftskritik artikuliert (Gayer/Engels 2011: 22; Engels/Gayer 2011: 39). In der Tat hat die feministische Friedensforscherin Tordis Batscheider angesichts der von ihr problematisierten Verstrickung der Friedensforschung in Politikberatung schon vor zwanzig Jahren einen Mangel an wissen- 
schaftstheoretischer Grundlagenforschung und Selbstreflexion festgestellt (Batscheider 1993). Eine Generation später schließe ich mich dieser Feststellung und Forderung gern an. Denn $»[\mathrm{k}]$ ritische Friedensforschung legitimiert sich dadurch, $\mathrm{da}[\mathrm{ss}]$ ihr wissenschaftliches Selbstverständnis mit der historisch-gesellschaftlichen Wirklichkeit in Wechselbeziehung steht und sie dabei ihren eigenen Standort im System organisierter Friedlosigkeit selbstkritisch reflektiert« (Batscheider 1993: 189). Eine zukünftige verstärkte Benennung und Bearbeitung des Verhältnisses zwischen politischer und epistemischer Gewalt sowie deren weitergehende Theoretisierung kann dazu einen sinnvollen Beitrag leisten.

\section{Literatur}

Alonso, Rogelio/Reinares, Fernando 2006: Maghreb Immigrants Becoming Suicide

Terrorists. A Case Study on Religious Radicalization Processes in Spain, in: Pedahzur 2006, 179-197.

Asad, Talal 2007: On Suicide Bombing, New York, NY. Atran, Scott 2003: Genesis of Suicide Terrorism, in: Science 299, 1534-1538.

Atran, Scott 2006: The Moral Logic and Growth of Suicide Terrorism, in: The Washington Quarterly 29: 2, 127-147.

Batscheider, Tordis 1993: Friedensforschung und Geschlechterverhältnis. Zur Begründung feministischer Fragestellungen in der kritischen Friedensforschung, Marburg.

Berko, Anat 2007: The Path to Paradise. The Inner World of Suicide Bombers and Their Dispatchers, Westport.

Biggs, Michael 2005: Dying Without Killing. Self-Immolations, 1963-2002, in: Gambetta 2005, 173-208.

Bloom, Mia 2005: Dying To Kill. The Allure of Suicide Terror, New York, NY.

Bourdieu, Pierre 1982: Die feinen Unterschiede. Kritik der gesellschaftlichen Urteilskraft, Frankfurt a.M.

Breckner, Roswitha 2007: Handout zum Verfahren der Segmentanalyse. (Unterlage zum Vortrag bei der Tagung »Soziologie des visuellen Wissens«, Wissenschaftszentrum Berlin, 25.05.2007), unv. Ms.

Brittain, Melisa 2006: Benevolent Invaders, Heroic Victims and Depraved Villains.

White Femininity in Media Coverage of the Invasion of Iraq, in: Hunt, Krista/ Rygiel, Kim (Hrsg.): (En)Gendering the War on Terror. War Stories and Camouflaged Politics, Aldershot, 73-96.

Brown, Wendy 2006: Regulating Aversion. Tolerance in the Age of Identity and Empire, Princeton, NJ. 
Brücher, Gertrud 2004: Postmoderner Terrorismus. Zur Neubegründung von Menschenrechten aus systemtheoretischer Perspektive, Opladen.

Brunner, Claudia 2007: Occidentalism Meets the Female Suicide Bomber. A Critical Reflection on Recent Terrorism Debates. A Review Essay, in: Signs. Journal of Women in Culture and Society 32: 4, 957-971.

Brunner, Claudia 2011 a: Wissensobjekt Selbstmordattentat. Epistemische Gewalt und okzidentalistische Selbstvergewisserung in der Terrorismusforschung, Wiesbaden.

Brunner, Claudia 2011 b: Geschlecht, Terrorismus, Wissenschaft. Reflexionen zum Verhältnis von politischer und epistemischer Gewalt am Beispiel des Wissensobjekts Selbstmordattentat, in: Engels, Bettina/Gayer, Corinna (Hrsg.): Neue Geschlechterperspektiven in der Friedens- und Konfliktforschung, Baden-Baden, 47-63.

Brunner, Claudia 2012 a: Un/Sichtbarkeiten im Terrorismuswissen. Die Sinnformel Selbstmordattentat auf Buchumschlägen der Terrorismusforschung, in: Keller, Reiner/Truschkat, Inga (Hrsg.): Anwendungen der Wissenssoziologischen Diskursanalyse, Wiesbaden, i.E.

Brunner, Claudia 2012 b: Unsterbliche Jungfrauen und paradiesische Zustände. Zur Historisierung von Selbstmordattentaten am Beispiel der Assassinen-Legende, in: Hikel, Christine/Schraut, Sylvia (Hrsg.): Terrorismus - Geschlecht - Erinnerung, Frankfurt a.M., i.E.

Bührmann, Andrea D./Schneider, Werner 2008: Vom Diskurs zum Dispositiv. Eine Einführung in die Dispositivanalyse, Bielefeld.

Collins, Patricia H. 1990: Black Feminist Thought. Knowledge, Consciousness, and the Politics of Empowerment, Sydney.

Coronil, Fernando 1996: Beyond Occidentalism. Toward Nonimperial Geohistorical Categories, in: Cultural Anthropology 11: 1, 51-87.

Coronil, Fernando 2000: Towards a Critique of Globalcentrism. Speculations on Capitalism's Nature, in: Public Culture 12: 2, 351-374.

Coronil, Fernando 2002: Jenseits des Okzidentalismus. Unterwegs zu nichtimperialen geohistorischen Kategorien, in: Conrad, Sebastian/Randeria, Shalini (Hrsg.): Jenseits des Eurozentrismus. Postkoloniale Perspektiven in den Geschichts- und Kulturwissenschaften, Frankfurt a.M, 177-218.

Coronil, Fernando 2009: Unterwegs zu einer Kritik des Globalzentrismus. Mutmaßungen über das Wesen des Kapitalismus, in: Dietze/Brunner/Wenzel 2009, $55-82$. 
Crenshaw, Kimberlé W. 1995: The Intersections of Race and Gender, in: Crenshaw, Kimberlé/Gotanda, Neil/Peller, Garry (Hrsg.): Critical Race Theory. The Key Writings That Formed the Movement, New York, NY, 357-383.

Cunningham, Karla J. 2003: Cross-Regional Trends in Female Terrorism, in: Studies in Conflict and Terrorism 26: 3, 171-195.

Dale, Stephen Frederic 1988: Religious Suicide in Islamic Asia. Anticolonial Terrorism in India, Indonesia, and the Philippines, in: Journal of Conflict Resolution 32: 1, 37-59.

Davis, Joyce M. 2003: Martyrs. Innocence, Vengeance, and Despair in the Middle East, New York, NY.

Dietze, Gabriele 2009: Okzidentalismuskritik. Möglichkeiten und Grenzen einer Forschungsperspektivierung, in: Dietze/Brunner/Wenzel 2009, 23-54.

Dietze, Gabriele/Brunner, Claudia/Wenzel, Edith (Hrsg.) 2009: Kritik des Okzidentalismus. Transdisziplinäre Beiträge $\mathrm{zu}$ (Neo-)Orientalismus und $\mathrm{Ge}-$ schlecht, Bielefeld.

Dolnik, Adam 2003: Die and Let Die. Exploring Links between Suicide Terrorism and Terrorist Use of Chemical, Biological, Radiological, and Nuclear Weapons, in: Studies in Conflict and Terrorism 26: 1, 17-35.

Duyvesteyn, Isabelle 2004: How New Is The New Terrorism?, in: Studies in Conflict and Terrorism 27: 5, 439-454.

Elshtain, Jean Bethke 1987: Women and War, New York, NY.

Elster, Jon 2005: Motivations and Beliefs in Suicide Missions, in: Gambetta 2005, 233-258.

Engels, Bettina/Gayer, Corinna 2011: Wie viel Feminismus soll es sein? Friedensund Konfliktforschung zwischen feministischer Theorie, Gender und Mainstream, in: Femina Politica. Zeitschrift für feministische Politikwissenschaft 20: $1,30-44$.

Enloe, Cynthia 1989: Bananas, Beaches and Bases. Making Feminist Sense of International Politics, London.

Enloe, Cynthia 2004: The Curious Feminist. Searching for Women in a New Age of Empire, Berkeley, CA.

Exo, Mechthild 2009: Die Gewalt der Konfliktforschung und Möglichkeiten des Widerstandes, in: Informationsstelle Militarisierung e.V. (Tagungsbericht IMIKongress 2009), in: http://www.imi-online.de/download/ME-KDoku-2009.pdf; 13.04.2011.

Fairclough, Norman 1995: Critical Discourse Analysis. The Critical Study of Language, London. 
Fegter, Susann 2011: Die Macht der Bilder. Photographien im Diskurs, in: Oelerich, Gertrud/ Otto, Hans-Uwe (Hrsg.): Soziale Arbeit und Empirische Forschung. Ein Studienbuch, Wiesbaden, 207-220.

Ferrero, Mario 2006: Martyrdom Contracts, in: Journal of Conflict Resolution 50: 6, 855-877.

Foucault, Michel 1978: Dispositive der Macht. Michel Foucault über Sexualität, Wissen und Wahrheit, Berlin.

Foucault, Michel 2000: Die >Gouvernementalität‘. Vorlesung am Collège de France 1977/1978, \Sécurité, territoire et population< vom 1. Februar 1978, in: Bröckling, Ulrich/Krasmann, Susanne/Lemke, Thomas (Hrsg.): Gouvernementalität der Gegenwart. Studien zur Ökonomisierung des Sozialen, Frankfurt a.M., 41-67.

Galtung, Johan 1975: Strukturelle Gewalt. Beiträge zur Friedens- und Konfliktforschung, Reinbek.

Gambetta, Diego (Hrsg.) 2005: Making Sense of Suicide Missions, New York, NY. Ganor, Boaz 2002 a: Suicide Attacks in Israel, in: ICT/ADL 2002, 140-152.

Ganor, Boaz 2002 b: Suicide Terrorism after September 11, in: ICT/ADL (Hrsg.): Countering Suicide Terrorism, Herzliya, 167-175.

Gayer, Corinna/Engels, Bettina 2011: Feministische Perspektiven in der Friedensund Konfliktforschung, in: dies. (Hrsg.): Geschlechterverhältnisse, Frieden und Konflikt. Feministische Denkanstöße für die Friedens- und Konfliktforschung, Baden-Baden, 9-26.

Geideck, Susan/Liebert, Wolf-Andreas 2003: Sinnformeln. Eine soziologisch-linguistische Skizze, in: dies. (Hrsg.): Sinnformeln. Linguistische und soziologische Analysen von Leitbildern, Metaphern und anderen kollektiven Orientierungsmustern, Berlin, 3-14.

Genette, Gérard 2001: Paratexte. Das Buch vom Beiwerk des Buches, Frankfurt a.M.

Glaser, Barney G./Strauss, Anselm L. 1998: Grounded Theory. Strategien qualitativer Forschung, Bern.

Hafez, Mohammed M. 2006: Dying to be Martyrs. The Symbolic Dimension of Suicide Terrorism, in: Pedahzur 2006, 54-80.

Hafez, Mohammed M. 2007: Suicide Bombers in Iraq. The Strategy and Ideology of Martyrdom, Washington.

Hagemann-White, Carol 2002: Gewalt im Geschlechterverhältnis als Gegenstand sozialwissenschaftlicher Forschung und Theoriebildung. Rückblick, gegenwärtiger Stand, Ausblick, in: Dackweiler, Regina-Maria/Schäfer, Reinhild (Hrsg.): 
Gewalt-Verhältnisse. Feministische Perspektiven auf Geschlecht und Gewalt, Frankfurt a.M., 29-52.

Hitzler, Ronald 2002: Sinnrekonstruktion. Zum Stand (in) der deutschsprachigen interpretativen Soziologie, in: Forum Qualitative Sozialforschung 3: 2, in: http:// www.qualitative-research.net/fqs-texte/2-02/2-02hitzler-d.htm; 13.05.2007.

Hoffman, Bruce 2002: Rethinking Terrorism and Counterterrorism Since 9/11, in: Studies in Conflict and Terrorism 25, 303-316.

Hoffman, Bruce 2003: The Logic of Suicide Terrorism, in: http://www.theatlantic.com/doc/200306/hoffman; 20.01.2006.

Hoffman, Bruce/McCormick, Gordon H. 2004: Terrorism, Signaling, and Suicide Attack, in: Studies in Conflict and Terrorism 27: 4, 243-281.

Holert, Tom 2004: Sicherheit, in: Bröckling, Ulrich/Krasmann, Susanne/Lemke,

Thomas (Hrsg.): Glossar der Gegenwart, Frankfurt a.M., 244-250.

Holmes, Stephen 2005: Al-Qaeda, September 11, 2001, in: Gambetta 2005, 131-172.

Hunt, Krista 2006: >Embedded Feminism < and the War on Terror, in: Hunt, Krista/

Rygiel, Kim (Hrsg.): (En)Gendering the War on Terror. War Stories and Camouflaged Politics, Aldershot, 57-71.

ICT (Hrsg.) 2001: Countering Suicide Terrorism. An International Conference, Herzliya.

ICT/ADL (Hrsg.) 2002: Countering Suicide Terrorism, Herzliya.

Israeli, Raphael 1997: Islamikaze and their Significance, in: Terrorism and Political Violence 9: 3, 96-121.

Israeli, Raphael 2003: Islamikaze. Manifestations of Islamic Martyrology, London. Jäger, Siegfried 1999: Kritische Diskursanalyse. Eine Einführung, Duisburg.

Juergensmeyer, Mark 2003: Terror in the Mind of God. The Global Rise of Religious Violence, Berkeley, CA.

Kajetzke, Laura 2008: Wissen im Diskurs. Ein Theorienvergleich von Bourdieu und Foucault, Wiesbaden.

Keller, Reiner 2004: Diskursforschung. Eine Einführung für SozialwissenschaftlerInnen, Opladen.

Keller, Reiner 2005: Wissenssoziologische Diskursanalyse. Grundlegung eines

Forschungsprogramms, Opladen.

Khosrokhavar, Farhad 2005: Suicide Bombers. Allah's New Martyrs. London.

Keller, Reiner/Truschkat, Inga (Hrsg.): Anwendungen der Wissenssoziologischen

Diskursanalyse. Theorie und Praxis der Diskursforschung, Wiesbaden, i.E.

Kimhi, Shaul/Even, Shemuel 2004: Who Are The Palestinian Suicide Bombers?, in:

Terrorism and Political Violence 16: 4, 815-840. 
Knapp, Gudrun-Axeli 2005: >Intersectionality< - ein neues Paradigma feministischer Theorie? Zur transatlantischen Reise von 〉Race, Class, Gender«, in: Feministische Studien 23: 1, 68-81.

Kress, Günther/van Leeuwen, Theo 1996: Reading Images. The Grammar of Visual Representation, London.

Kushner, Harvey 1996: Suicide Bombers. Business as Usual, in: Studies in Conflict and Terrorism 19: 4, 329-337.

Laqueur, Walter 2003: No End to War. Terrorism in the Twenty-First Century, New York, NY.

Lester, David/Yang, Bijou/Lindsay, Mark 2004: Suicide Bombers. Are Psychological Profiles Possible?, in: Studies in Conflict and Terrorism 27: 4, 283-295.

Lewis, Bernard 2002: What Went Wrong?, in: The Atlantic Monthly 289: 1, in: http://www.theatlantic.com/doc/prem/200201/lewis; 25.04.2007.

Lewis, Jeffrey W. 2007: Precision Terror. Suicide Bombing as Control Technology, in: Terrorism and Political Violence 19: 2, 223-245.

Lewis, Reina/Mills, Sara (Hrsg.) 2003: Feminist Postcolonial Theory. A Reader, Edinburgh.

Loew, Peter Oliver 2006: Polen auf dem Buch. Die Umschlaggestaltung deutschsprachiger Veröffentlichungen über Polen (Vortrag bei der Tagung »Visuelle Erinnerungskulturen und Geschichtskonstruktionen in Deutschland und Polen«, 29.09.2006), Darmstadt.

Mamdani, Mahmood 2005: Good Muslim, Bad Muslim. America, The Cold War, and The Roots of Terror, New York, NY.

McCall, Leslie 2005: The Complexity of Intersectionality, in: Signs. Journal of Women in Culture and Society 30: 3, 1771-1800.

McGee, Michael Calvin 1980: The >Ideograph $<$. A Link Between Rhetoric and Ideology, in: Quarterly Communication Monographs 50, 47-65.

Merari, Ariel 1990: The Readiness to Kill and Die. Suicidal Terrorism in the Middle East, in: Reich, Walter (Hrsg.): Origins of Terrorism. Psychologies, Ideologies, Theologies, States of Mind, Cambridge, 192-207.

Moghadam, Assaf 2003: Palestinian Suicide Terrorism in the Second Intifada. Motivations and Organizational Aspects, in: Studies in Conflict and Terrorism 26: 2, 65-92.

Moghadam, Assaf 2006 a: Defining Suicide Terrorism, in: Pedahzur 2006, 13-24.

Moghadam, Assaf 2006 b: The Roots of Suicide Terrorism. A Multi-Causal Approach, in: Pedahzur 2006, 81-107.

Morgan, Mary S./Morrison, Margaret (Hrsg.) 1999: Models as Mediators. Perspectives on Natural and Social Science, Cambridge. 
Nagel, Sarah 2009: Hochschulen forschen für den Krieg, in: Informationsstelle Militarisierung e.V. (Tagungsbericht IMI-Kongress 2009), in: http://imi-online.de/ download/SN-Juni2009-Hochschulen.pdf; 13.04.2011.

O'Neill, Richard 1981: Suicide Squads. Axis and Allied Special Attack Weapons of World War II. Their Development and Their Missions, London.

Ó Tuathail, Gearóid 1996: Critical Geopolitics. The Politics of Writing Global Space, London.

Pape, Robert A. 2003: The Strategic Logic of Suicide Terrorism, in: American Political Science Review 97: 3, 343-361.

Pape, Robert A. 2005: Dying to Win. The Strategic Logic of Suicide Terrorism, New York, NY.

Pappe, Ilan 2011: Wissenschaft als Herrschaftsdienst. Der Kampf um die akademische Freiheit in Israel, Hamburg.

Pedahzur, Ami 2004: Toward an Analytical Model of Suicide Terrorism - A Comment, in: Terrorism and Political Violence 16: 4, 841-844.

Pedahzur, Ami 2005: Suicide Terrorism, Cambridge.

Pedahzur, Ami (Hrsg.) 2006: Root Causes of Suicide Terrorism. The Globalization of Martyrdom, Abingdon.

Randeria, Shalini 1999: Geteilte Geschichte und verwobene Moderne, in: Rüsen, Jörn/Leitgeb, Hanna/Jegelka, Norbert (Hrsg.): Zukunftsentwürfe. Ideen für eine Kultur der Veränderung, Frankfurt a.M., 87-96.

Regener, Susanne 2006: Visuelle Kultur, in: Ayaß, Ruth/Bergmann, Jörg R. (Hrsg.): Qualitative Methoden in der Medienforschung, Reinbek, 435-355.

Reich, Walter (Hrsg.) 1990: Origins of Terrorism. Psychologies, Ideologies, Theologies, States of Mind, Cambridge.

Reuter, Christoph 2004: My Life Is a Weapon. A Modern History of Suicide Bombing, Princeton, NJ.

Ricolfi, Luca 2005: Palestinians, 1981-2003, in: Gambetta 2005, 77-129.

Said, Edward 1978: Orientalism, London.

Sarasin, Philipp 2003: Geschichtswissenschaft und Diskursanalyse, Frankfurt a.M. Schweitzer, Yoram 2002: Suicide Terrorism. Development and Main Characteristics, in: ICT/ADL 2002, 77-88.

Schweitzer, Yoram 2006: Al-Qaeda and the Global Epidemic of Suicide Attacks, in: Pedahzur 2006, 132-151.

Shay, Shaul 2004: The Shahids. Islam and Suicide Attacks, New Brunswick, NJ. Silke, Andrew 2006: The Role of Suicide in Politics, Conflict, and Terrorism, in:

Terrorism and Political Violence 18: 1, 35-46.

Skaine, Rosemarie 2006: Female Suicide Bombers, Jefferson, NC. 
Spivak, Gayatri C. 1988: Can the Subaltern Speak?, in: Carry, Nelson/Grossberg, Lawrence (Hrsg.): Marxism and the Interpretation of Culture, Urbana, IL, 271-313.

Spivak, Gayatri C. 2008: Can the Subaltern Speak? Postkolonialität und subalterne Artikulation, Wien.

Sprinzak, Ehud 2000: Rational Fanatics, in: Foreign Policy, Sep/Oct 2000, 66-73. Stern, Jessica 2003: Terror in the Name of God. Why Religious Militants Kill, New York, NY.

Strenski, Ivan 2003: Sacrifice, Gift and the Social Logic of Muslim >Human Bombers $<$, in: Terrorism and Political Violence 15: 3, 1-34.

Sylvester, Christine 2002: Feminist International Relations. An Unfinished Journey, Cambridge.

Thayer, Bradley A./Hudson, Valerie M. 2010: Sex and the Shaheed. Insights from the Life Sciences on Islamic Suicide Terrorism, in: International Security Studies 34: 4, 37-62.

Titscher, Stefan/Wodak, Ruth/Meyer, Michael/Vetter, Eva 1998: Methoden der Textanalyse. Leitfaden und Überblick, Opladen.

Victor, Barbara 2004: Army of Roses. Inside the World of Palestinian Women Suicide Bombers, London.

Walgenbach, Katharina/Dietze, Gabriele/Hornscheidt, Antje/Palm, Kerstin 2007: Gender als interdependente Kategorie. Neue Perspektiven auf Intersektionalität, Diversität und Heterogenität, Opladen.

Weinberg, Leonard 2006: Suicide Terrorism for Secular Causes, in: Pedahzur 2006, 108-121.

Weinberg, Leonard/Pedahzur, Ami/Canetti-Nisim, Daphna 2003: The Social and Religious Characteristics of Suicide Bombers and Their Victims, in: Terrorism and Political Violence 15: 3, 139-153.

Whitworth, Sandra 1997: Feminist International Relations. Towards a Political

Economy of Gender in Interstate and Non-Governmental Institutions, Houndmills.

Yeğenoğlu, Meyda 1999: Colonial Fantasies. Towards a Feminist Reading of Orientalism, Cambridge.

\section{Die Autorin}

Dr. phil. Claudia Brunner, Universitätsassistentin, Zentrum für Friedensforschung und Friedenspädagogik, Alpen-Adria-Universität Klagenfurt

E-Mail: claudia.brunner@uni-klu.ac.at 